\title{
Behavioral health integration model
}

\author{
Sanjay Basu, MD, PhD ${ }^{1,2}$, John W. Williams Jr, MD, MHS ${ }^{3}$, and Russell S. Phillips, MD ${ }^{2}$ \\ ${ }^{1}$ Center for Population Health Sciences and Center for Primary Care and Outcomes Research, Departments of Medicine and of Health Research \\ and Policy, Stanford University, Stanford, CA, USA; ${ }^{2}$ Center for Primary Care, Harvard Medical School, Boston, MA, USA; ${ }^{3}$ Departments of Medicine \\ and Psychiatry, Duke University School of Medicine and Center of Innovation for Health Services Research in Primary Care, Durham VA Health \\ System, Durham, NC, USA.
}

J Gen Intern Med 33(10): 1597

DOI: $10.1007 / \mathrm{s} 11606-018-4512-9$

(c) Society of General Internal Medicine 2018

$\mathrm{W}$ e appreciate Dr. Tynan's letter but respectfully disagree with some of his contentions. First, our CoCM model simulation was not purely telephone based, having included components of in-person evaluation, follow-up, and referral when needed. CoCM studies do have primarily telephonebased intervention but allow for face-to-face interactions, and our simulation matched the Medicare implementation guidelines for telephone and face-to-face visit frequency. ${ }^{2}, 3$ Our staffing metrics were similarly concordant with actual implementation; per the University of Washington's Advancing Integrated Mental Health Solutions (AIMS) center, "a fulltime behavioral health care manager typically oversees 100 150 patients," and our psychiatrist consult time was additionally based on real-world implementation experience and varied widely in Monte Carlo simulations to reflect practice variations. ${ }^{4}, 5$

Second, the typical duration of sessions in the referenced literature and in our model are concordant. Visit durations in the cited literature ranged from 25 to $60 \mathrm{~min}^{3}$, and we simulated an additional hour for initial visits and half hour each for subsequent visits for administrative, documentation, and discussion time (again, matching Medicare rules).

Third, we believe it would be unfair to increase time estimates for CoCM beyond those for typical implementation, while minimizing the costs of PCBH to those reflecting its theoretical minimal implementation rather typical implementation. Economic modeling of care implementation requires discernment of resource staffing and reimbursement for typical costs and reimbursements, not the theoretically minimal costs.

Performing rigorous financial models is important to provide objective comparisons from scholars with genuine equipoise between approaches, but we note these approaches are not mutually exclusive, as both CoCM and PCBM have been blended in practice. Financing of behavioral healthcare in the current fee-for-service environment is a critical barrier to

Published online June 18, 2018 adoption, and its financial viability is directly the target of Medicare's policy change, which prompted our analysis. Reductions in emergency room visits and re-hospitalizations have unfortunately not been consistently demonstrated from these approaches, despite being theoretically possible and desirable, and do not directly influence a primary care practices' ability to finance behavioral healthcare. We have argued for a move away from fee-for-service towards capitated payment approaches, but nevertheless the fee-for-service environment is only starting to become more value-based. Objective fiscal calculations have thus been desired to avoid an overly optimistic view of actual financing needs, which may lead to under-funding of practices by third-party payers.

Corresponding Author: Sanjay Basu, $M D, P h D$; Center for Primary Care Harvard Medical School, Boston, MA, USA (e-mail: basus@stanford.edu).

\section{Compliance with Ethical Standards:}

Conflict of Interest: The authors declare that they do not have a conflict of interest.

\section{REFERENCES}

1. Dietrich AJ, Oxman TE, Williams JW, et al. Re-engineering systems for the treatment of depression in primary care: cluster randomised controlled trial. BMJ. 2004;329(7466):602. doi:https://doi.org/10.1136/bmj.38219. 481250.55

2. Centers for Medicare and Medicaid Services. Fact Sheet Behavioral Health Integration Services. Medicare Learning Network. https://www.cms.gov/ Outreach-and-Education / Medicare-Learning-Network-MLN / MLNProducts/Downloads/BehavioralHealthIntegration.pdf. Published 2018.

3. Centers for Medicare and Medicaid Services. FAQs for billing the Psychiatric Collaborative Care Management ( CoCM ) codes ( G0502-G0504) and General Behavioral Health Intervention ( BHI ) code ( G0507) FAQs for billing the Psychiatric Collaborative Care Management ( CoCM ) codes ( G0502-G0504 ) a. https://www.psychiatry.org/File Library/Psychiatrists/ Practice/Professional-Topics/Integrated-Care/FAQs-Billing-PsychiatricCollaborative-Care-Management-Codes.pdf\%0D\%0A. Published 2017.

4. University of Washington AIMS Center. Guidelines on Caseload Size for Behavioral Health Care Managers and Psychiatric Consultants. aims.uw. edu/sites/default/files/CareManager_CaseloadSize_Guidelines.pdf. Published 2017.

5. University of Washington AIMS Center. Behavioral Health Care Manager. http://aims.uw.edu/collaborative-care/team-structure/care-manager. Accessed April 13, 2018. 